\title{
Study of the Relationship Between School Managers' Communicative Skills and Schools' Atmosphere
}

\author{
Özdemir Ali ${ }^{1,2} \&$ Getmez Demiray ${ }^{2}$ \\ ${ }^{1}$ Department of Educational Administration, Marmara University, Istanbul, Turkey \\ ${ }^{2}$ Ministry of Education, Istanbul, Turkey \\ Correspondence: Özdemir Ali, Ph.D. Assistant Professor, Department of Educational Administration, Marmara \\ University, Ataturk Faculty of Education Goztepe Campus 34722, Kadıkoy, Istanbul, Turkey. E-mail: \\ aliozdmr32@gmail.com
}

Received: January 2, 2019 Accepted: February 1, 2019 Online Published: February 25, 2019

doi:10.5539/jel.v8n2p145 URL: https://doi.org/10.5539/jel.v8n2p145

\begin{abstract}
Schools are places where social interactions take place highest. Teachers, students and parents are the individuals of school management that constitute this social life place. A school's success is closely related with its workers' fulfilling the duties that are expected from them within an interaction. Every school has its own unique atmosphere. Many factors play an important role for this atmosphere to emerge, however the most important of these is communication skills. Hence for a school's expected success to be accomplished a strong communication must be created. In that sense a school manager's communicative skill is an important factor for a school's success to be accomplished.

In this research, school managers' communicative skills on schools' atmospheres are studied among the communication processes that exist in schools. Decreasing the problems to minimum that stem from communication, what school mangers can and may do to improve their communicative skills are explained, discussed and ordered in this research.

The aim of this research is to determine the effects of school managers' communication skills on a school's atmosphere. Relational screening model among quantitative methods were used in this research. Target population of the study consists of teachers and school manager assistans commisioned in official state schools in Istanbul from the districts of Sultanbeyli, Tuzla and Pendik. In this target population, total of 378 teachers constituted the sampling group, determined through random selection method.
\end{abstract}

Keywords: education, management, school atmosphere, communication

\section{Introduction}

Since the time humanity has started to exist, they relied on various organizations to accomplish specific ends. To sustain social needs and resolve the problems that emerge in a society, people need to cooperate and conduct activities together with the other members of the society. As the natural result of this, orginizations emerge (Aydin, 1993). Organizations consist of parties that create the society. When society's need has an increment about something and hen works need to be done deftly, individuals inside the organization organize the individuals inside it to create units to accomplish the work/job in a qualified way. Every social unit which is created is an organization (Başaran, 2008, p. 15).

An atmosphere which emerges in an organization is the result of communication between the individuals and groups in that organization. The ration between of an organization's targets being actualized and its individuals expectations being met affects its clitmate. Schools are public places where several social interactions occur. While school managers, teachers and other personnels who work in the school perform their duties, the interaction and communication they form must be strong. Unless a school management has an effective communication process with teachers, other personnels and parents, it may lead to some negativity in school. Furthermore aforementioned siutation may cause the school to fail which is a social living space. In that sense it can be said that interpersonal relations and communications have important roles in schools (Bursalığlu, 2010).

In an organization, behaviors of the members of an organization and their competency of communication are influential on the atmosphere of the organization (Bursalığlu, 2005, pp. 24-25). Bursalığlu considers one of 
school managers' competency fields as organizational atmosphere in one of his researches which is about "educational manager's competencies".

Teachers say that when they move from one school to another, from the first moments they feel various characteristics of the new school which they move to. Some of these characteristics are visible to the eye. For instance; decoration of the school, the architectural features of the school, wall writings, its cleanness etc... Moreoever varios characteristics of the school are noticed right away; for example, positive affair of the school personnel towards each other, school administration's authority's presence, teachers' methods and techniques, school personnels' commitment to the school etc... These differences that exist affect the workers, students and parents of that school. This interaction which takes place in a school create the atmosphere of that school (Wei, 2003, p. 2).

School's atmosphere is the sum of characteristics that makes a school different than the others, it has an effect on a school's personnel's behavior and it creates the social and psychological atmosphere. A school manager is the one who is the determinant of a school's organizational atmosphere. School managers may contribute to school in terms of increasing school personnels' motivation and creating a healthy school culture (Çelik, 2005, p. 45).

Many researches that are conducted indicate that a school's atmosphere affects many fields of a school and its personnels. In schools which have positive atmosphere, it is seen that students have less behavioral and emotional problems (Kuperminc, Leadbeater, Emmons ve Blatt, 1997, p. 76). A school's atmosphere is understood through the school's work conditions and these conditions' effects on its workers. A school atmosphere which occurs in a school affects the motivations of its workers, their senses of belonging, morales and work performances. Thus first of all, school managers must create a school atmosphere which must affect a school's personnels in a positive way (Şişman, 2004, p. 101).

Hoy's (2003) School Atmosphere:

1) School's physical characteristics

2) School personnels' previous cultural experiences and gender differences

3) Quality of human affairs that take place in a school

4) Common values and beliefs which are created by school personnel.

To create a positive atmosphere in a school, what are expected of school managers are as follows (Bursalıoğlu, 1982):

1) Being able to maintain balance between a school's goals and its workers' expectations.

2) Being able to apply the punishment and reward systems in a way that increases efficiency and motivation.

3) School manager must use his/her effectiveness before he/she uses their authority.

4) To sustain the possibilities of security and protection of the school in return of school workers' commitment to the school.

5) To give priority of applying the rules which are taken together with the workers of the school.

To accomplish the goals and aims of a shool, it is a fundamental condition of a organizational positive atmosphere to occur (Balc1,1993, p. 31). The most important factor which affects a school's success is the effectiveness of the processes of communication which take places in that school. A school manager's being able to accomplish the goals and aims of a school, his/her being able to state those aims and goals and being able to convey these aims and goals to the personnels of the school are possible through the skill of communication. The communicative competencies which are among a school manager's must-have fundamental competencies have important impact on other personnels' attitudes and behaviors which are necessary for them to fulfill their duties (Çelik, 2007, p. 3). 3 of the fundamental features among the features which a school manager must have are: knowledge of management, fundamental knowledge of the field which he/she works in and skill and knowledge of communication. Among these 3 fundamental features, knowledge of management and level of necessity of fundamental knowledge for the field may vary depending on the position of the manager. On the other hand, the view that communicative skills being the same level for every managers in different positions and all knowledge and skills of management must be nearly at 50\% level is an accepted approach (Açıkalın, 1995, p. 39).

The features that an effective school manager must have may be listed as follows: being able to use the mother tongue effectively and correctly, having an effective persuasion, being effective at interpersonal communications, having a skillful listening, having a strong written and oral communication skills (Şahin, 2000). In a research which is conducted to determine the competencies that are needed in school managers who work at primary 
schools, it was determined that communication must be a fundamental skills of management (Şahin, 2000).

School principals spend most of their time communicating with school staff (Başaran, 1993, p. 70). One of the main characteristics of a successful school principal is to have effective communication skills (Deas, 1994, p. 44; Geddes, 1995, p. 1). An effective school principal must be in constant contact with the teachers. An effective school principal must have strong, written-verbal communication and persuasion skills (Şişman, 2012, pp. 144146). In order for school principals to initiate and maintain an effective communication process, some communication elements, communication principles and strategies; they should have skills in eliminating communication barriers and organizing communication processes (Şimşek, 1997, p. 97; Bursalığlu, 1999, p. 42). It is important to have communication channels open in defining and solving many problems in schools (Özden, 2013, p. 88).

In school, which is the basic element of education system, the very basic duty of a school manager is to accomplish the aims and goals that are set in a school and respond the expectations of the personnel of the school. For a school manager to be able to accomplish the goals and aims of a school, he/she must utilize monatery and human resources of school efficiently. In that sense for a school manager to reach these goals that are set, besides knowing the concepts and processes of the educational management, he/she must is expected to have effective communication skills and the ability to create a positive atmosphere in school. Thus, in this research an answer to the question of "whether there is correlation between a school manager's communicative skills and a school's atmosphere" is sought.

\section{Method}

\subsection{Research Design}

In this research it is aimed to assess communication skills's effect on a school's atmosphere. Relational screening model was used of quantitative methods. Relational screening, which is a version of the survey model, is a model that aims to determine the presence of variation and/or its level among numerous variables. Relational screening model has two types, one being correlational and the other being comparative: While in methods which are corellational types, whether variables change together in relation with each other and how do the change occur are studied, in the comparative styled models, whether there is difference between groups in relation to dependant variable are studied by creating groups according to independent variable (Karasar, 1995, pp. 81-82).

\subsection{Target Population and Sample}

Target population of the study consists of teachers and school manager assistans commisioned in official state schools in Istanbul from the districts of Sultanbeyli, Tuzla and Pendik. In this target population, total of 378 teachers constituted the sampling group, determined through random selection method.

The fundamental features of random sampling methods are their robustness in terms their samples' representing the target population and units, which are taken as basis in sampling, having equal probabilities of getting chosen for the sample. With these methods, it is aimed to form samples which have strong representations that can make valid generalizations for the target population (Büyüköztürk vd, 2009).

\subsection{Data Collection Tool and Application}

For the collection of the data from the teachers and school managers from the target population, "Personal Information Request form", "Scale for Communicative Competency" (Wiemann, 1977), "Scale for School Atmosphere" (Canl, 2016) were used.

The data which were acquired from scales were coded by means of computer and resolved through the packet program SPSS 22. For the first part of the scale frequency and percentage distribution was done. To determine the managers' perceptions arithmetic mean, standard deviation were done. For determining the differences between attitudes, the paired comparison was normal, thus "t" test was used one-way analysis of variance (ANOVA) was used to compare groups with more than two variables. In case the differance was found significant, Scheffe (Multiple Comparison) and Mann Whitney U test was applied. Whether there were significant differences between views was tested at a significance level of $a<0.05$.

\subsubsection{Personal Information Request Form}

The questionnaire which is formed to determine the demographic information of the teachers and school managers. In the form quesions, variables such as state of managing (school manager assistant)", gender, seniority, graduation, school manager's age exist. 


\subsubsection{Communicative Competence Scale}

The scale was develeloped by Wiemann (1977). The communicative competency scale of Wiemann originally consists of 36 articles and 5 subdimensions. Originally the scale is made of grading of 5 points. The subdimensions in the scale consist of (general communicative Competence)(1., 5. , 6. , 9. , 20. , 34. \& 23. articles), competency of emphaty (10. , 12. , 13. , 14. , 15. , 17.\& 24. articles), competence of affiliation/support (7. , 8. , 18. , 22. , $30 ., 33$. articles), competency of behavioral flexibility (2., 3. , 19., 31. , 36. articles), competency of social relaxation (29. , 32. , 26. , 16. , 35. articles). 4. , 11., 21., 25. , 27. and 28 articles that are in the scale are not in the subdimensions of the scale.

Wiemann (1977) calculated the validity and reliability of the structural reliability with factor analysis and for reliability's calculation in subdimensions he calculated Cronbach's Alpha quotients in internal-reliability. According to this he found the scale's Cronbach's Alpha as=96.

\subsubsection{Scale of School Atmosphere}

"School Climate Scale“ developed by Canlı (2016). The School Atmosphere Scale developed by Live consists of 23 items and 5 sub-dimensions.

Each item in the school climate scale is based on a Likert-type scale consisting of " $1=$ Never", " $2=$ Rarely", "3=Sometimes", "4=Mostly", "5=Always".

The sub-dimensions of the school atmosphere are:

1) The aspects of Being Democratic and dedication to school $(1,2,3,4,5,6)$.

2) Leadership and interaction aspect $(7,8,9,10,11,12)$.

$3)$ Factors of success $(13,14,15,16)$.

4) Sincerity aspect $(17,18,19)$.

5) Conflict aspect $(20,21,22,23)$ articles.

As a result of the analyzes conducted to determine the internal consistency of the items in the scale, Cronbach Alpha internal consistency coefficient is for democratic and school dedication. 908 and for the aspects of leadership and interaction 90.897 , for the aspects of success factors 89.753 , "for the aspects of sincerity". 852 and for the aspects of the conflict 85.730 . Based on the findings obtained in this context, it can be said that the scale is valid and reliable.

\subsubsection{Collection of the Data}

In the academic year 2016-2017, after the sample was determined and the necessary permissions for the research were taken through legal means, the schools were contacted with the teachers and assistant managers and the necessary information was given to the schools. The scales were multiplied and applied by the number of teachers and deputy directors. Analysis was carried out on 378 people.

\section{Results}

\subsection{Recruitment}

The universe of the research consists of teachers and deputy principals who work in official schools in Sultanbeyli, Tuzla and Pendik districts of Istanbul. A total of 378 teachers were selected from this population by random sampling. 


\subsection{Participant Flow}

Table 1. Frequency and percentage values of the participants' demographic information

\begin{tabular}{|c|c|c|c|}
\hline & Groups & $f$ & $\%$ \\
\hline \multirow[t]{2}{*}{ Position } & Teacher & 349 & 94.1 \\
\hline & Assistant Manager & 22 & 5.9 \\
\hline \multirow[t]{2}{*}{ Gender } & Male & 148 & 39.9 \\
\hline & Female & 223 & 60.1 \\
\hline \multirow[t]{3}{*}{ Education } & College & 16 & 4.3 \\
\hline & Undergraduate & 332 & 89.5 \\
\hline & Master's Degree & 23 & 6.2 \\
\hline \multirow[t]{4}{*}{ Seniority } & $1-5$ year(s) & 123 & 33.2 \\
\hline & $6-10$ years & 107 & 28.8 \\
\hline & $11-15$ years & 81 & 21.8 \\
\hline & 16 years and above & 60 & 16.2 \\
\hline \multirow[t]{11}{*}{ Field } & Form Teacher & 200 & 53.9 \\
\hline & Turkish & 22 & 5.9 \\
\hline & Mathematic & 32 & 8.6 \\
\hline & Science and Tech. & 31 & 8.4 \\
\hline & Social Studies & 19 & 5.1 \\
\hline & Foreign Language & 21 & 5.7 \\
\hline & Religious Culture & 13 & 3.5 \\
\hline & Visual Art & 8 & 2.2 \\
\hline & Music & 7 & 1.9 \\
\hline & Physical Education & 6 & 1.6 \\
\hline & Counseling & 12 & 3.2 \\
\hline \multirow[t]{4}{*}{ School Manager's Age } & 39 years and below & 105 & 28.3 \\
\hline & $40-49$ years & 184 & 49.6 \\
\hline & 50 years and above & 82 & 22.1 \\
\hline & Total & 371 & 100.0 \\
\hline
\end{tabular}

As shown in Table 1, 349 (94.1\%) of the 371 teachers who make up the sample were teachers and $22(5.9 \%)$ were manager's assistants. $148(38.9 \%)$ were male and $223(60.1 \%)$ were female. 16 of them $(4.3 \%)$ with college, 332 of them (89.5\%) with bachelor's degree, 23 of them $(6.2 \%)$ with graduate degree, 123 of them were $(33.2 \%)$ of $1-$ 5 years, 107 (28.8\%), 6-10 years, $81(21.8 \%) 11-15$ years, and $60(16.2 \%)$ have 16 years or more seniority. 200 (53.9\%) classroom teachers, 22 (5.9\%) Turkish, 32 (8.6\%) mathematics, 31 (8.4\%) science and technology and remaining $23 \%$ are from different branches. The age of school managers which are in number of $105(28.3 \%)$ aged 39 and under, 184 of them were (49.6) between $40-49$ years old, 82 of them (22.1\%) were over 50 years of age.

\subsection{Statistics and Data Analysis}

The level of communication qualifications perceived by teachers in school managers is given in Table 2.

Table 2. Arithmetic mean, standard deviation values of total scores and all sub-dimensions of communication competencies scale

\begin{tabular}{llll}
\hline Aspect & $\mathrm{N}$ & $\overline{\mathrm{X}}$ & $\mathrm{Ss}$ \\
\hline Aspect of General Communicative Competency & 371 & 25,81 & 4,129 \\
Aspect of Emphaty Competency & 371 & 29,27 & 4,675 \\
Aspect of Affilitiaton/Support Competency & 371 & 21,35 & 3,154 \\
Aspect of Behavioral Flexibilty Competency & 371 & 17,93 & 2,475 \\
Aspect of Socialrelaxation Competency & 371 & 12,74 & 2,108 \\
General Scale of Communication & 371 & 107,11 & 15,230 \\
\hline
\end{tabular}

As shown in Table 2, the Communication Proficiency Scale of the teachers who make up the sample group is the General Communication Adequacy Size scores ( $\mathrm{X}=25,81, \mathrm{ss}=4,129)$, and the Empathy Competency Aspect scores ( $\bar{X}=29,27, s s=4,675)$. Supporting Competency Aspect scores ( $\bar{X}=21,35, \mathrm{ss}=3,154)$, Behavioral Flexibility Competency Aspect scores $(\mathrm{X}=17,93$, ss $=2,475)$, Social Comfort Competency Aspect scores $(\bar{X}=12,74$, 
ss $=2,108)$, General Communication Scale scores $(\bar{X}=107,11, \mathrm{ss}=15,230)$ were observed. Based on the arithmetic averages, the level of communication proficiencies that teachers perceive in school principals, most of the time in the dimension of general communication is as follows: general communicative competency as "most of the time", Competency of Emphaty as "most of the time", Affiliation and support as "most of the time", Behavioral Flexibility Competence as "most of the time", Social Comfort Competence as "sometimes".

Evaluation of General Communication Levels of Teachers in School Principals by Various Demographic Variables:

The levels of General Communication that teachers perceive in school managers; differentiation analysis related to age, gender, educational status, seniority and age of school principal are given below.

Table 3. General communication levels of teachers perceived by school principals according to their duties Mann Whitney u test results

\begin{tabular}{|c|c|c|c|c|c|c|c|}
\hline Point & Position & $N$ & $\bar{x}_{\text {sira }}$ & $\sum_{\text {sira }}$ & $U$ & $z$ & $p$ \\
\hline \multirow[t]{3}{*}{ GIYB } & Teacher & 349 & 185,96 & 64901,00 & 3826,000 &,- 027 & ,979 \\
\hline & Manager's Assistant & 22 & 186,59 & 4105,00 & & & \\
\hline & Total & 371 & & & & & \\
\hline \multirow[t]{3}{*}{ EYB } & Teacher & 349 & 185,56 & 64760,50 & 3685,500 &,- 316 & ,752 \\
\hline & Manager's Assistant & 22 & 192,98 & 4245,50 & & & \\
\hline & Total & 371 & & & & & \\
\hline \multirow[t]{3}{*}{ BDYB } & Öğretmen & 349 & 186,16 & 64970,00 & 3783,000 &,- 116 & ,908 \\
\hline & Manager's Assistant & 22 & 183,45 & 4036,00 & & & \\
\hline & Total & 371 & & & & & \\
\hline \multirow[t]{3}{*}{ EDYB } & Teacher & 349 & 185,21 & 64638,00 & 3563,000 &,- 585 & ,559 \\
\hline & Manager's Assistant & 22 & 198,55 & 4368,00 & & & \\
\hline & Total & 371 & & & & & \\
\hline \multirow[t]{3}{*}{ SRYB } & Teacher & 349 & 185,01 & 64568,50 & 3493,500 &,- 720 & ,471 \\
\hline & Manager's Assistant & 22 & 201,70 & 4437,50 & & & \\
\hline & Toplam & 371 & & & & & \\
\hline \multirow[t]{3}{*}{ General Scale } & Teacher & 349 & 185,52 & 64748,00 & 3673,000 &,- 340 & ,734 \\
\hline & Manager's assistant & 22 & 193,55 & 4258,00 & & & \\
\hline & Total & 371 & & & & & \\
\hline
\end{tabular}

As shown in Table 3, the teachers were applied the Mann Whitney U Test to determine the difference between the levels of general communication that they perceived in school managers according to their duties.

Table 4 . The general communication levels of teachers perceived by school managers according to their gender $t$ test results

\begin{tabular}{|c|c|c|c|c|c|c|c|c|}
\hline \multirow[t]{2}{*}{ Point } & \multirow[t]{2}{*}{ Gender } & \multirow[t]{2}{*}{$N$} & \multirow[t]{2}{*}{$\bar{X}$} & \multirow[t]{2}{*}{$S S$} & \multirow[t]{2}{*}{$\mathrm{Sh}_{\overline{\mathrm{x}}}$} & \multicolumn{3}{|l|}{$t$ Test } \\
\hline & & & & & & $t$ & $S d$ & $p$ \\
\hline \multirow[t]{2}{*}{ GIYYB } & Male & 148 & 26,13 & 4,058 & ,334 & 1,216 & 369 & ,225 \\
\hline & Female & 223 & 25,60 & 4,171 & ,279 & & & \\
\hline \multirow[t]{2}{*}{ EYB } & Male & 148 & 29,72 & 4,530 & ,372 & 1,493 & 369 & , 136 \\
\hline & Female & 223 & 28,98 & 4,756 & ,318 & & & \\
\hline \multirow[t]{2}{*}{ BDYB } & Male & 148 & 21,68 & 3,102 & ,255 & 1,635 & 369 & ,103 \\
\hline & Female & 223 & 21,13 & 3,176 & ,213 & & & \\
\hline \multirow[t]{2}{*}{ EDYB } & Male & 148 & 18,06 & 2,469 & ,203 & ,812 & 369 & ,417 \\
\hline & Female & 223 & 17,85 & 2,481 & , 166 & & & \\
\hline \multirow[t]{2}{*}{ SRYB } & Male & 148 & 13,07 & 1,883 & ,155 & 2,425 & 369 & ,016 \\
\hline & Female & 223 & 12,53 & 2,224 & , 149 & & & \\
\hline \multirow[t]{2}{*}{ Genel Ölçek } & Male & 148 & 108,65 & 15,025 & 1,235 & 1,594 & 369 & ,112 \\
\hline & Female & 223 & 106,08 & 15,313 & 1,025 & & & \\
\hline
\end{tabular}

As shown in Table 4, the independent group t-test was used to determine the difference between the general communication levels perceived by the school principals according to their gender and there was no significant difference in the total scale and other sub-dimensions. General Communication As seen in Table 3, independent 
group t Test was performed to determine the difference between the general communication levels perceived by school managers according to their genders and no significant difference was found between the total scale and other sub-dimensions while the Social Comfort Competence Dimension was found to be significant. In the dimension of Social Comfort Competence, it was observed that men evaluated school principals more positively than women.

Table 5. General communication levels of school principals according to their education Kruskal Wallis $\mathrm{h}$ test results

\begin{tabular}{|c|c|c|c|c|c|}
\hline Point & Education & $N$ & $\bar{x}_{\text {sira }}$ & $x^{2}$ & $p$ \\
\hline \multirow[t]{4}{*}{ GIYB } & College & 16 & 191,22 &, 051 & ,975 \\
\hline & Undergraduate & 332 & 185,61 & & \\
\hline & Master's Degree & 23 & 187,98 & & \\
\hline & Total & 371 & & & \\
\hline \multirow[t]{4}{*}{ EYB } & College & 16 & 201,63 & 1,324 &, 516 \\
\hline & Undergraduate & 332 & 183,83 & & \\
\hline & Master's Degree & 23 & 206,48 & & \\
\hline & Total & 371 & & & \\
\hline \multirow[t]{4}{*}{ BDYB } & College & 16 & 186,09 &, 711 & ,701 \\
\hline & Undergraduate & 332 & 187,25 & & \\
\hline & Master's Degree & 23 & 167,89 & & \\
\hline & Total & 371 & & & \\
\hline \multirow[t]{4}{*}{ EDYB } & College & 16 & 215,22 & 1,448 & ,485 \\
\hline & Undergraduate & 332 & 185,19 & & \\
\hline & Master's Degree & 23 & 177,37 & & \\
\hline & Total & 371 & & & \\
\hline \multirow[t]{4}{*}{ SRYB } & College & 16 & 231,41 & 3,643 &, 162 \\
\hline & Undergraduate & 332 & 182,87 & & \\
\hline & Master's Degree & 23 & 199,63 & & \\
\hline & Total & 371 & & & \\
\hline \multirow[t]{4}{*}{ General Scale } & College & 16 & 200,56 & ,352 & 838 \\
\hline & Undergraduate & 332 & 185,03 & & \\
\hline & Master's Degree & 23 & 189,87 & & \\
\hline & Total & 371 & & & \\
\hline
\end{tabular}

As seen in Table 5, according to the education of teachers, Kruskal Wallis $\mathrm{h}$ Test was used to determine the difference between the general communication levels perceived in school managers and no significant difference was found with the total scale in any sub-dimension. 
Table 6. General communication levels of school principals according to seniority of teachers' Anova test results

\begin{tabular}{|c|c|c|c|c|c|c|c|c|c|c|}
\hline \multicolumn{5}{|c|}{$f, \quad \overline{\mathrm{X}}$ ve SS Values } & \multicolumn{6}{|c|}{ ANOVA Results } \\
\hline Puan & Kidem & $N$ & $\overline{\mathrm{x}}$ & ss & Var. K. & $K T$ & $S d$ & $\mathrm{KO}$ & $F$ & $p$ \\
\hline \multirow[t]{5}{*}{ GIYB } & $1-5$ year(s) & 123 & 26,54 & 3,302 & G. Between & 223,480 & 3 & 74,493 & 4,494 & ,004 \\
\hline & $6-10$ years & 107 & 26,09 & 3,899 & G. In & 6083,933 & 367 & 16,577 & & \\
\hline & $11-15$ years & 81 & 25,46 & 4,345 & Total & 6307,412 & 370 & & & \\
\hline & 16 years and above & 60 & 24,28 & 5,260 & & & & & & \\
\hline & Total & 371 & 25,81 & 4,129 & & & & & & \\
\hline \multirow[t]{5}{*}{ EYB } & $1-5$ year(s) & 123 & 30,21 & 3,544 & G. Between & 292,358 & 3 & 97,453 & 4,589 &, 004 \\
\hline & $6-10$ years & 107 & 29,61 & 4,570 & G. In & 7793,146 & 367 & 21,235 & & \\
\hline & $11-15$ years & 81 & 28,42 & 4,674 & Total & 8085,504 & 370 & & & \\
\hline & 16 years and above & 60 & 27,90 & 6,243 & & & & & & \\
\hline & Total & 371 & 29,27 & 4,675 & & & & & & \\
\hline \multirow[t]{5}{*}{ BDYB } & $1-5$ year(s) & 123 & 21,93 & 2,459 & G. Between & 197,129 & 3 & 65,710 & 6,924 &, 000 \\
\hline & $6-10$ years & 107 & 21,79 & 2,722 & G. In & 3483,016 & 367 & 9,491 & & \\
\hline & $11-15$ years & 81 & 20,90 & 3,277 & Total & 3680,146 & 370 & & & \\
\hline & 16 years and above & 60 & 19,95 & 4,320 & & & & & & \\
\hline & Total & 371 & 21,35 & 3,154 & & & & & & \\
\hline \multirow[t]{5}{*}{ EDYB } & $1-5$ year(s) & 123 & 18,20 & 2,036 & G. Between & 77,607 & 3 & 25,869 & 4,336 & 005 \\
\hline & $6-10$ years & 107 & 18,21 & 2,206 & G. In & 2189,708 & 367 & 5,967 & & \\
\hline & $11-15$ years & 81 & 17,89 & 2,455 & Total & 2267,315 & 370 & & & \\
\hline & 16 years and above & 60 & 16,93 & 3,409 & & & & & & \\
\hline & Total & 371 & 17,93 & 2,475 & & & & & & \\
\hline \multirow[t]{5}{*}{ SRYB } & $1-5$ year(s) & 123 & 12,96 & 1,862 & G. Between & 22,852 & 3 & 7,617 & 1,724 &, 162 \\
\hline & $6-10$ years & 107 & 12,90 & 1,990 & G. In & 1621,822 & 367 & 4,419 & & \\
\hline & $11-15$ years & 81 & 12,53 & 2,197 & Total & 1644,674 & 370 & & & \\
\hline & 16 years and above & 60 & 12,32 & 2,581 & & & & & & \\
\hline & Total & 371 & 12,74 & 2,108 & & & & & & \\
\hline General & $1-5 \mathrm{y} 11$ & 123 & 109,85 & 11,244 & G. Between & 3424,298 & 3 & 1141,433 & 5,084 & 002 \\
\hline \multirow[t]{4}{*}{ Scale } & $6-10$ years & 107 & 108,61 & 14,157 & G. In & 82402,602 & 367 & 224,530 & & \\
\hline & $11-15$ years & 81 & 105,20 & 15,620 & Toplam & 85826,900 & 370 & & & \\
\hline & 16 years and above & 60 & 101,38 & 21,079 & & & & & & \\
\hline & Total & 371 & 107,11 & 15,230 & & & & & & \\
\hline
\end{tabular}

As shown in Table 6, the ANOVA test was used to determine the difference between the level of general communication perceived of the school managers according to teacher' seniority and there was no significant difference in Aspect of Social Comfort Competence. Aspect of Empathy Competence, Aspect of Affiliation and Supporting Competence, Aspect of Behavioral Flexibility Competence, Aspect of Social Comfort Competence, General Communication Scale. Scheffe's test results are given in Table 7. 
Table 7. Scheffe test results

\begin{tabular}{|c|c|c|c|c|c|}
\hline Aspect & Seniority(i) & Seniority(j) & $\bar{x}_{i}-\bar{x}_{i}$ & $S h_{\bar{x}}$ & $P$ \\
\hline \multirow[t]{12}{*}{ GIYYB } & \multirow[t]{3}{*}{$1-5$ year(s) } & $6-10$ yil & ,443 & 538 & ,878 \\
\hline & & $11-15$ y1l & 1,080 & ,583 & ,331 \\
\hline & & 16 yıl ve üz & $2,253^{*}$ & 641 &, 007 \\
\hline & \multirow[t]{3}{*}{$6-10$ years } & $1-5 \mathrm{y} 11$ &,- 443 &, 538 &, 878 \\
\hline & & $11-15$ y1l & ,637 & 600 &, 771 \\
\hline & & 16 yıl ve üz & 1,810 & 657 &, 057 \\
\hline & \multirow[t]{3}{*}{$11-15$ years } & $1-5$ yil & $-1,080$ & ,583 & ,331 \\
\hline & & $6-10$ yıl &,- 637 & ,600 & ,771 \\
\hline & & 16 yıl ve üz & 1,173 & ,694 & ,414 \\
\hline & \multirow[t]{3}{*}{16 years and above } & $1-5$ year(s) & $-2,253^{*}$ & 641 & 007 \\
\hline & & $6-10$ years & $-1,810$ & ,657 &, 057 \\
\hline & & $11-15$ years & $-1,173$ & 694 &, 414 \\
\hline \multirow[t]{12}{*}{ EYB } & \multirow[t]{3}{*}{$1-5$ year(s) } & $6-10$ years & ,604 & 609 &, 805 \\
\hline & & $11-15$ years & 1,792 & ,659 &, 062 \\
\hline & & 16 years and above & $2,311^{*}$ &, 726 & 018 \\
\hline & \multirow[t]{3}{*}{$6-10$ years } & $1-5$ year $(\mathrm{s})$ &,- 604 & 609 &, 805 \\
\hline & & $11-15$ years & 1,188 & 679 &, 383 \\
\hline & & 16 years and above & 1,707 & ,743 &, 155 \\
\hline & \multirow[t]{3}{*}{$11-15$ years } & $1-5$ year $(\mathrm{s})$ & $-1,792$ & 659 &, 062 \\
\hline & & $6-10$ years & $-1,188$ & 679 &, 383 \\
\hline & & 16 years and above &, 520 & ,785 & ,932 \\
\hline & \multirow[t]{3}{*}{16 years amd above } & $1-5$ year $(\mathrm{s})$ & $-2,311^{*}$ &, 726 &, 018 \\
\hline & & $6-10$ years & $-1,707$ &, 743 &, 155 \\
\hline & & $11-15$ years &,- 520 & ,785 & ,932 \\
\hline \multirow[t]{12}{*}{ BDYB } & \multirow[t]{3}{*}{$1-5$ year(s) } & $6-10$ years & ,141 & ,407 & ,989 \\
\hline & & $11-15$ years & 1,034 & ,441 &, 141 \\
\hline & & 16 years and above & $1,985^{*}$ & 485 & 001 \\
\hline & \multirow[t]{3}{*}{$6-10$ years } & $1-5$ year $(\mathrm{s})$ &,- 141 &, 407 & ,989 \\
\hline & & $11-15$ years &, 893 & ,454 & 277 \\
\hline & & 16 years and above & $1,844^{*}$ & ,497 &, 004 \\
\hline & \multirow[t]{3}{*}{$11-15$ years } & $1-5$ year(s) & $-1,034$ &, 441 &, 141 \\
\hline & & $6-10$ years &,- 893 &, 454 & ,277 \\
\hline & & 16 years and above & ,951 &, 525 &, 351 \\
\hline & \multirow[t]{3}{*}{16 years and above } & $1-5$ year(s) & $-1,985^{*}$ & 485 & 001 \\
\hline & & $6-10$ years & $-1,844^{*}$ &, 497 &, 004 \\
\hline & & $11-15$ years &,- 951 &, 525 & 351 \\
\hline \multirow[t]{12}{*}{ EDYB } & $1-5$ year(s) & $6-10$ years &,- 012 &, 323 & 1,000 \\
\hline & & $11-15$ years &, 314 &, 350 &, 847 \\
\hline & & 16 years and above & $1,270^{*}$ &, 385 & 013 \\
\hline & $6-10$ years & $1-5$ year $(\mathrm{s})$ &, 012 &, 323 & 1,000 \\
\hline & & $11-15$ years &, 326 &, 360 &, 844 \\
\hline & & 16 years and above & $1,282^{*}$ &, 394 &, 015 \\
\hline & $11-15$ years & $1-5$ year $(\mathrm{s})$ &,- 314 &, 350 &, 847 \\
\hline & & $6-10$ years &,- 326 &, 360 &, 844 \\
\hline & & 16 years and above &, 956 &, 416 &, 155 \\
\hline & 16 years and above & $1-5$ year(s) & $-1,270^{*}$ &, 385 & 013 \\
\hline & & $6-10$ years & $-1,282^{*}$ &, 394 &, 015 \\
\hline & & $11-15$ years &,- 956 & ,416 &, 155 \\
\hline General & $1-5$ year(s) & $6-10$ year(s) & 1,238 & 1,981 & ,942 \\
\hline Scale & & $11-15$ years & 4,648 & 2,144 & 197 \\
\hline & & 16 years and above & $8,462^{*}$ & 2,360 &, 005 \\
\hline & $6-10$ years & $1-5$ year $(\mathrm{s})$ & $-1,238$ & 1,981 & ,942 \\
\hline & & $11-15$ years & 3,410 & 2,207 & ,497 \\
\hline & & 16 years and above & $7,224^{*}$ & 2,417 &, 031 \\
\hline & $11-15$ years & $1-5$ year $(\mathrm{s})$ & $-4,648$ & 2,144 & ,197 \\
\hline & & $6-10$ years & $-3,410$ & 2,207 &, 497 \\
\hline & & 16 years and above & 3,814 & 2,552 &, 526 \\
\hline & 16 years and above & $1-5$ year $(\mathrm{s})$ & $-8,462^{*}$ & 2,360 &, 005 \\
\hline & & $6-10$ years & $-7,224^{*}$ & 2,417 &, 031 \\
\hline & & $11-15$ years & $-3,814$ & 2,552 &, 526 \\
\hline
\end{tabular}

As shown in Table 7, according to the seniority of teachers' perception of managers' level of communication competency, difference between the groups of general communication Scheffe test was done and the test result, the difference in the total scale of 1-5 years seniority and those who have a seniority of 6-10 years those with less than 
16 years of age and those with seniority have been found to be more positive for school managers. The same results were observed in all sub-dimensions.

Table 8. Teachers' level of perception about general communication levels of school managers' Anova test results

\begin{tabular}{|c|c|c|c|c|c|c|c|c|c|c|}
\hline \multicolumn{5}{|c|}{$f$, $\mathrm{x}$ ve $s s$ Values } & \multicolumn{6}{|c|}{ ANOVA Results } \\
\hline Point & Age & $\mathrm{N}$ & $\bar{x}$ & Ss & Var. K. & $K T$ & $S d$ & $K O$ & $\mathrm{~F}$ & $P$ \\
\hline \multirow[t]{4}{*}{ GIYB } & 39 years and below & 105 & 26,10 & 3,734 & G. Between & 429,445 & 2 & 214,722 & 13,443 & 000 \\
\hline & $40-49$ years & 184 & 26,53 & 3,511 & G. In & 5877,968 & 368 & 15,973 & & \\
\hline & 50 years and above & 82 & 23,82 & 5,179 & Total & 6307,412 & 370 & & & \\
\hline & Total & 371 & 25,81 & 4,129 & & & & & & \\
\hline \multirow[t]{4}{*}{ EYB } & 39 years and below & 105 & 29,65 & 4,183 & G. Between & 362,112 & 2 & 181,056 & 8,627 &, 000 \\
\hline & $40-49$ years & 184 & 29,88 & 4,063 & G. In & 7723,392 & 368 & 20,987 & & \\
\hline & 50 years and above & 82 & 27,43 & 5,965 & Total & 8085,504 & 370 & & & \\
\hline & Total & 371 & 29,27 & 4,675 & & & & & & \\
\hline \multirow[t]{4}{*}{ BDYB } & 39 years and below & 105 & 21,79 & 2,752 & G. Between & 266,677 & 2 & 133,338 & 14,375 & 000 \\
\hline & $40-49$ years & 184 & 21,80 & 2,668 & G. In & 3413,469 & 368 & 9,276 & & \\
\hline & 50 years ad above & 82 & 19,76 & 4,042 & Total & 3680,146 & 370 & & & \\
\hline & Total & 371 & 21,35 & 3,154 & & & & & & \\
\hline \multirow[t]{4}{*}{ EDYB } & 39 years and below & 105 & 18,14 & 2,363 & G. Between & 185,140 & 2 & 92,570 & 16,361 &, 000 \\
\hline & $40-49$ years & 184 & 18,40 & 1,911 & G. In & 2082,176 & 368 & 5,658 & & \\
\hline & 50 years and above & 82 & 16,62 & 3,207 & Total & 2267,315 & 370 & & & \\
\hline & Total & 371 & 17,93 & 2,475 & & & & & & \\
\hline \multirow[t]{4}{*}{ SRYB } & 39 years and below & 105 & 13,02 & 1,787 & G. Between & 115,877 & 2 & 57,939 & 13,947 & 000 \\
\hline & $40-49$ years & 184 & 13,05 & 1,813 & G. In & 1528,796 & 368 & 4,154 & & \\
\hline & 50 years and above & 82 & 11,70 & 2,711 & Total & 1644,674 & 370 & & & \\
\hline & Total & 371 & 12,74 & 2,108 & & & & & & \\
\hline General & 39 years and below & 105 & 108,70 & 13,072 & G. Between & 6446,188 & 2 & 3223,094 & 14,942 &, 000 \\
\hline \multirow[t]{3}{*}{ Scale } & $40-49$ years & 184 & 109,66 & 12,521 & G. In & 79380,712 & 368 & 215,708 & & \\
\hline & 50 years and above & 82 & 99,32 & 20,159 & Total & 85826,900 & 370 & & & \\
\hline & Total & 371 & 107,11 & 15,230 & & & & & & \\
\hline
\end{tabular}

As shown in Table 8, the ANOVA test was used to determine the difference between the level of general communication perceived in the school managers according to teachers' seniority and there was no significant difference in Social Comfort Competence Dimension. 
Table 9. Scheffe test results

\begin{tabular}{|c|c|c|c|c|c|}
\hline Aspect & Year (i) & Years (j) & $\bar{x}_{i}-\bar{x}_{i}$ & $S h_{\bar{x}}$ & $P$ \\
\hline \multirow[t]{6}{*}{ GİYB } & \multirow[t]{2}{*}{39 years and below } & $40-49$ years &,- 422 & 489 & 689 \\
\hline & & 50 years and above & $2,288^{*}$ &, 589 &, 001 \\
\hline & \multirow[t]{2}{*}{$40-49$ years } & 39 years and below &, 422 & ,489 & ,689 \\
\hline & & 50 years and above & $2,710^{*}$ &, 531 &, 000 \\
\hline & \multirow[t]{2}{*}{50 yeasr and above } & 39 years and below & $-2,288^{*}$ &, 589 &, 001 \\
\hline & & $40-49$ years & $-2,710^{*}$ &, 531 &, 000 \\
\hline \multirow[t]{6}{*}{ EYB } & \multirow[t]{2}{*}{39 years and below } & $40-49$ years &,- 233 &, 560 & ,917 \\
\hline & & 50 yeasrs and above & $2,221^{*}$ & 675 &, 005 \\
\hline & \multirow[t]{2}{*}{$40-49$ years } & 39 years and below &, 233 &, 560 & ,917 \\
\hline & & 50 years and above & $2,454^{*}$ & 608 &, 000 \\
\hline & \multirow[t]{2}{*}{50 years and above } & 39 years and below & $-2,221^{*}$ & 675 &, 005 \\
\hline & & $40-49$ years & $-2,454^{*}$ & ,608 &, 000 \\
\hline \multirow[t]{6}{*}{ BDYB } & \multirow[t]{2}{*}{39 years and below } & $40-49$ years &,- 014 &, 372 & ,999 \\
\hline & & 50 years and above & $2,034^{*}$ & ,449 &, 000 \\
\hline & \multirow[t]{2}{*}{$40-49$ years } & 39 yaş ve altı &, 014 & ,372 & ,999 \\
\hline & & 50 yaş ve üz & $2,048^{*}$ &, 404 &, 000 \\
\hline & \multirow[t]{2}{*}{50 years and above } & 39 yaş ve altı & $-2,034^{*}$ & ,449 &, 000 \\
\hline & & 40-49 yaş & $-2,048^{*}$ & ,404 &, 000 \\
\hline \multirow[t]{6}{*}{ EDYB } & \multirow[t]{2}{*}{39 years and below } & 40-49 yaş &,- 254 & ,291 & ,684 \\
\hline & & 50 yaş ve üz & $1,521^{*}$ &, 351 &, 000 \\
\hline & \multirow[t]{2}{*}{$40-49$ years } & 39 yaş ve altı &, 254 & ,291 & 684 \\
\hline & & 50 yaş ve üz & $1,775^{*}$ &, 316 &, 000 \\
\hline & \multirow[t]{2}{*}{50 years and above } & 39 years and below & $-1,521^{*}$ &, 351 &, 000 \\
\hline & & $40-49$ years & $-1,775^{*}$ &, 316 &, 000 \\
\hline \multirow[t]{6}{*}{ SRYB } & \multirow[t]{2}{*}{39 years ve above } & $40-49$ years &,- 035 & 249 & ,990 \\
\hline & & 50 years and above & $1,324^{*}$ & ,300 &, 000 \\
\hline & \multirow[t]{2}{*}{$40-49$ years } & 39 years and below &, 035 & ,249 & ,990 \\
\hline & & 50 years and above & $1,359^{*}$ & 271 &, 000 \\
\hline & \multirow[t]{2}{*}{50 years and above } & 39 years and below & $-1,324^{*}$ & 300 &, 000 \\
\hline & & $40-49$ years & $-1,359^{*}$ & 271 &, 000 \\
\hline \multirow[t]{6}{*}{ Genel Ölçek } & \multirow[t]{2}{*}{39 years and below } & $40-49$ years &,- 958 & 1,796 & ,867 \\
\hline & & 50 years and above & $9,388^{*}$ & 2,164 &, 000 \\
\hline & \multirow[t]{2}{*}{$40-49$ years } & 39 years and below & ,958 & 1,796 & ,867 \\
\hline & & 50 years and above & $10,346^{*}$ & 1,950 &, 000 \\
\hline & \multirow[t]{2}{*}{50 years and above } & 39 years and below & $-9,388^{*}$ & 2,164 &, 000 \\
\hline & & $40-49$ years & $-10,346^{*}$ & 1,950 &, 000 \\
\hline
\end{tabular}

As shown in Table 9, the Scheffe test was used to determine the difference between the groups of general communication levels of the school managers depending on the ages of the school managers. It is observed that the individuals who have less have evaluated the school principals more positively. The same results were observed in all sub-dimensions.

School Atmosphere Levels According to Teachers' Perceptions.

According to teachers' perceptions, school climate scale levels are given in Table 10.

Table 10. School atmosphere total points and arithmetic mean, standard deviation values of all sub-dimensions

\begin{tabular}{llll}
\hline Aspect & N & $\overline{\mathrm{x}}$ & Ss \\
\hline Aspects of Being Democratic and Dedication to School & 371 & 25,47 & 3,576 \\
Aspects of Leadership and Interaction & 371 & 25,61 & 4,034 \\
Aspect of Success Factors & 371 & 17,05 & 2,377 \\
Aspect of Sincerity & 371 & 11,93 & 2,171 \\
Aspect of Conflict & 371 & 8,99 & 3,401 \\
General Scale & 371 & 89,06 & 9,142 \\
\hline
\end{tabular}

As shown in Table 10, it was found that the scores of the school's atmosphere scale according to the perceptions of the teachers of the sample group were Being Democratic and Dedication to school aspect scores ( $x=25.47$, 
$\mathrm{ss}=3.576)$, and the Leadership and Interaction aspect scores $(\bar{x}=25.61, \mathrm{ss}=4.034)$, Success Factors aspect scores $(\bar{x}=17,053, s s=2,377)$, Sincerity Aspect scores $(\bar{x}=11,93, s s=2,171)$, Conflict Aspect scores $(\bar{x}=8,99, s s=3,401)$, Total Scale scores $(\mathrm{x}=89,06, \mathrm{ss}=9,142)$. According to teachers' perceptions, school atmosphere levels are as follows: Being Democratic and Dedication Aspect to School is "always", Leadership and Interaction Aspects as "always", Aspects of Factors of Success as "always", Sincerity Aspect is as "most of the time", Conflict Aspect is as "Rarely".

Evaluation of School Atmosphere's Perceptions of Teachers According to Various Demographic Variables, differentiation analysis related to age, gender, educational status, seniority and age of school principal are given below.

Table 11. Mann Whitney u test results of school climate perceptions according to teachers' position

\begin{tabular}{llllllll}
\hline Point & Position & $N$ & $\bar{x}_{\text {sira }}$ & $\sum_{\text {sira }}$ & $U$ & $z$ & $p$ \\
\hline DOAB & Teacher & 349 & 184,76 & 64479,50 & 3404,500 &,- 896 &, 370 \\
& Manager assistant & 22 & 205,75 & 4526,50 & & & \\
& Total & 371 & & & & & \\
\multirow{2}{*}{ LEB } & Teacher & 349 & 183,59 & 64071,50 & 2996,500 & $-1,741$ &, 082 \\
& Manager assistant & 22 & 224,30 & 4934,50 & & & \\
& Total & 371 & & & & & \\
BEB & Teacher & 349 & 184,72 & 64467,50 & 3392,500 &,- 927 & \\
& Manager assistant & 22 & 206,30 & 4538,50 & & & \\
& Total & 371 & & & & & \\
SB & Teacher & 349 & 182,49 & 63690,50 & 2615,500 & $-2,548$ & \\
& Manager assistant & 22 & 241,61 & 5315,50 & & & \\
& Total & 371 & & & & & \\
ÇB & Teacher & 349 & 184,89 & 64528,00 & 3453,000 &,- 795 & \\
& Manager assistant & 22 & 203,55 & 4478,00 & & & \\
& Total & 371 & & & & & \\
\multirow{2}{*}{ General Scale } & Teacher & 349 & 183,57 & 64065,50 & 2990,500 & $-1,740$ & \\
& Manager assistant & 22 & 224,57 & 4940,50 & & & \\
& Total & 371 & & & & & \\
\hline
\end{tabular}

As shown in Table 11, Mann Whitney U Test was used to determine the differences between school atmosphere's perceptions of teachers according to their duties.

In the dimension of intimacy, the deputy principals evaluated the school atmosphere more positively than the teachers.

Table 12. T test results of teachers' perceptions of school in terms of their genders

\begin{tabular}{|c|c|c|c|c|c|c|c|c|}
\hline \multirow[t]{2}{*}{ Point } & \multirow[t]{2}{*}{ Gender } & \multirow[t]{2}{*}{$N$} & \multirow[t]{2}{*}{$\overline{\mathrm{X}}$} & \multirow[t]{2}{*}{$S S$} & \multirow[t]{2}{*}{$\mathrm{Sh}_{\overline{\mathrm{x}}}$} & \multicolumn{3}{|l|}{$t$ Test } \\
\hline & & & & & & $t$ & $S d$ & $p$ \\
\hline \multirow[t]{2}{*}{ DOAB } & Male & 148 & 25,66 & 3,681 & ,303 & ,806 & 369 & ,421 \\
\hline & Female & 223 & 25,35 & 3,507 & ,235 & & & \\
\hline \multirow[t]{2}{*}{ LEB } & Male & 148 & 26,05 & 3,895 & ,320 & 1,698 & 369 & ,090 \\
\hline & Female & 223 & 25,32 & 4,106 & ,275 & & & \\
\hline \multirow[t]{2}{*}{ BEB } & Male & 148 & 17,06 & 2,405 & , 198 & ,081 & 369 & ,935 \\
\hline & Female & 223 & 17,04 & 2,364 & , 158 & & & \\
\hline \multirow[t]{2}{*}{ SB } & Male & 148 & 11,73 & 2,329 & ,191 & $-1,469$ & 369 & ,143 \\
\hline & Female & 223 & 12,07 & 2,053 & ,138 & & & \\
\hline \multirow[t]{2}{*}{ ÇB } & Male & 148 & 8,70 & 3,275 & ,269 & $-1,348$ & 369 & ,178 \\
\hline & Female & 223 & 9,19 & 3,476 & ,233 & & & \\
\hline \multirow[t]{2}{*}{ General Scale } & Male & 148 & 89,20 & 9,562 & ,786 &, 234 & 369 &, 815 \\
\hline & Female & 223 & 88,97 & 8,873 &, 594 & & & \\
\hline
\end{tabular}

As shown in Table 12, $\mathrm{T}$ test was performed to determine the differences between school atmoshpere perceptions of teachers according to their gender and no significant difference was found between the total scale and other sub-dimensions. 
Table 13. Kruskal Wallis H Test results of school atmosphere perceptions of teachers according to their education

\begin{tabular}{|c|c|c|c|c|c|}
\hline Point & Education & $N$ & $\bar{x}_{\text {sira }}$ & $x^{2}$ & $p$ \\
\hline \multirow{4}{*}{ DOAB } & College & 16 & 192,63 & 1,721 &, 423 \\
\hline & Undergraduate & 332 & 183,78 & & \\
\hline & Master's Degree & 23 & 213,37 & & \\
\hline & Total & 371 & & & \\
\hline \multirow[t]{4}{*}{ LEB } & College & 16 & 229,69 & 3,269 & , 195 \\
\hline & Undergraduate & 332 & 183,04 & & \\
\hline & Master's Degree & 23 & 198,39 & & \\
\hline & Total & 371 & & & \\
\hline \multirow[t]{4}{*}{ BEB } & Yüksek Okul & 16 & 208,16 & 3,156 & ,206 \\
\hline & Undergraduate & 332 & 182,70 & & \\
\hline & Master's Degree & 23 & 218,22 & & \\
\hline & Total & 371 & & & \\
\hline \multirow[t]{4}{*}{ SB } & Yüksek Okul & 16 & 204,56 & 5,660 & 059 \\
\hline & Undergraduate & 332 & 181,82 & & \\
\hline & Master's Degree & 23 & 233,43 & & \\
\hline & Total & 371 & & & \\
\hline \multirow[t]{4}{*}{ ÇB } & Yüksek Okul & 16 & 202,75 &, 532 &, 767 \\
\hline & Undergraduate & 332 & 185,76 & & \\
\hline & Master's Degree & 23 & 177,78 & & \\
\hline & Total & 371 & & & \\
\hline \multirow[t]{4}{*}{ General Scale } & Yüksek Okul & 16 & 217,44 & 3,043 & ,218 \\
\hline & Undergraduate & 332 & 182,69 & & \\
\hline & Master's Degree & 23 & 211,96 & & \\
\hline & Total & 371 & & & \\
\hline
\end{tabular}

As shown in Table 13, the Kruskal Wallis $\mathrm{H}$ Test was used to determine the differences between school atmosphere perceptions of teachers according to their education and no significant difference was found between the total scale and other sub-dimensions.

Table 14. Anova test results of school atmosphere perceptions by teachers' seniorities

\begin{tabular}{|c|c|c|c|c|c|c|c|c|c|c|}
\hline \multicolumn{5}{|c|}{$\overline{f, \quad \bar{X}}$ ve SS Values } & \multicolumn{6}{|c|}{ ANOVA Results } \\
\hline Point & Seniority & $N$ & $\bar{x}$ & ss & Var. K. & $K T$ & $S d$ & $K O$ & $F$ & $p$ \\
\hline \multirow[t]{5}{*}{$\overline{\mathrm{DOAB}}$} & $1-5$ year(s) & 123 & 25,24 & 3,622 & G. Between & 34,982 & 3 & 11,661 & 911 & ,436 \\
\hline & $6-10$ years & 107 & 25,94 & 3,247 & G. In & 4695,471 & 367 & 12,794 & & \\
\hline & $11-15$ years & 81 & 25,25 & 3,430 & Total & 4730,453 & 370 & & & \\
\hline & 16 years and above & 60 & 25,42 & 4,192 & & & & & & \\
\hline & Total & 371 & 25,47 & 3,576 & & & & & & \\
\hline \multirow[t]{5}{*}{ LEB } & $1-5$ year(s) & 123 & 25,79 & 3,926 & G. Between & 44,548 & 3 & 14,849 & 912 & ,435 \\
\hline & $6-10$ years & 107 & 25,96 & 3,762 & G. In & 5975,560 & 367 & 16,282 & & \\
\hline & $11-15$ years & 81 & 25,07 & 3,859 & Total & 6020,108 & 370 & & & \\
\hline & 16 years and above & 60 & 25,35 & 4,878 & & & & & & \\
\hline & Total & 371 & 25,61 & 4,034 & & & & & & \\
\hline \multirow[t]{5}{*}{ BEB } & $1-5$ year(s) & 123 & 17,11 & 2,371 & G. Between &, 817 & 3 & ,272 & ,048 & ,986 \\
\hline & $6-10$ years & 107 & 17,02 & 2,603 & G. In & 2090,310 & 367 & 5,696 & & \\
\hline & $11-15$ years & 81 & 16,99 & 2,353 & Total & 2091,127 & 370 & & & \\
\hline & 16 years and above & 60 & 17,07 & 2,033 & & & & & & \\
\hline & Total & 371 & 17,05 & 2,377 & & & & & & \\
\hline \multirow[t]{5}{*}{ SB } & $1-5$ yıl & 123 & 11,87 & 2,150 & G. Between & 18,498 & 3 & 6,166 & 1,312 &, 270 \\
\hline & $6-10$ y1l & 107 & 12,27 & 2,086 & G. In & 1724,817 & 367 & 4,700 & & \\
\hline & $11-15$ y1l & 81 & 11,73 & 2,056 & Total & 1743,315 & 370 & & & \\
\hline & 16 yıl ve üz & 60 & 11,73 & 2,476 & & & & & & \\
\hline & Toplam & 371 & 11,93 & 2,171 & & & & & & \\
\hline \multirow[t]{5}{*}{ ÇB } & $1-5$ yıl & 123 & 9,16 & 3,077 & G. Between & 5,312 & 3 & 1,771 & ,152 & ,928 \\
\hline & $6-10$ yıl & 107 & 8,89 & 3,175 & G. In & 4274,677 & 367 & 11,648 & & \\
\hline & $11-15$ y1l & 81 & 8,94 & 3,607 & Total & 4279,989 & 370 & & & \\
\hline & 16 yıl ve üz & 60 & 8,92 & 4,139 & & & & & & \\
\hline & Toplam & 371 & 8,99 & 3,401 & & & & & & \\
\hline General & $1-5$ yıl & 123 & 89,16 & 8,490 & G. Between & 228,771 & 3 & 76,257 & ,912 & ,435 \\
\hline \multirow[t]{4}{*}{ Scale } & $6-10$ y1l & 107 & 90,08 & 8,316 & G. In & 30693,925 & 367 & 83,635 & & \\
\hline & $11-15$ y1l & 81 & 87,98 & 9,286 & Toplam & 30922,695 & 370 & & & \\
\hline & 16 yıl ve üz & 60 & 88,48 & 11,402 & & & & & & \\
\hline & Toplam & 371 & 89,06 & 9,142 & & & & & & \\
\hline
\end{tabular}


As shown in Table 14, ANOVA Test was used to determine the differences between school climate perceptions of teachers according to their seniority and no significant difference was found in the total scale other sub-dimensions.

Table 15. Anova test results of school climate perceptions by teachers' age

\begin{tabular}{|c|c|c|c|c|c|c|c|c|c|c|}
\hline \multicolumn{5}{|c|}{$f, \mathrm{x}$ ve ss Values } & \multicolumn{6}{|c|}{ ANOVA Results } \\
\hline Point & Groups & $\mathrm{N}$ & $\bar{x}$ & Ss & Var. K. & $K T$ & $S d$ & $K O$ & $\mathrm{~F}$ & $P$ \\
\hline \multirow[t]{4}{*}{ DOAB } & 39 years amd below & 105 & 25,19 & 4,017 & G. Between & 55,273 & 2 & 27,637 & 2,175 &, 115 \\
\hline & $40-49$ years & 184 & 25,85 & 3,167 & G. in & 4675,180 & 368 & 12,704 & & \\
\hline & 50 years and above & 82 & 24,98 & 3,787 & Total & 4730,453 & 370 & & & \\
\hline & Total & 371 & 25,47 & 3,576 & & & & & & \\
\hline \multirow[t]{4}{*}{ LEB } & 39 years and below & 105 & 25,41 & 4,090 & G. Between & 188,418 & 2 & 94,209 & 5,945 & 003 \\
\hline & $40-49$ years & 184 & 26,24 & 3,459 & G. in & 5831,690 & 368 & 15,847 & & \\
\hline & 50 years and above & 82 & 24,45 & 4,846 & Total & 6020,108 & 370 & & & \\
\hline & Total & 371 & 25,61 & 4,034 & & & & & & \\
\hline \multirow[t]{4}{*}{ BEB } & 39 years and below & 105 & 16,78 & 2,777 & G. Between & 14,262 & 2 & 7,131 & 1,264 & ,284 \\
\hline & $40-49$ years & 184 & 17,23 & 2,113 & G. in & 2076,864 & 368 & 5,644 & & \\
\hline & 50 years and above & 82 & 16,98 & 2,378 & Total & 2091,127 & 370 & & & \\
\hline & Total & 371 & 17,05 & 2,377 & & & & & & \\
\hline \multirow[t]{4}{*}{ SB } & 39 years and below & 105 & 11,60 & 2,352 & G. Between & 16,519 & 2 & 8,259 & 1,760 & , 173 \\
\hline & $40-49$ years & 184 & 12,09 & 2,052 & G. in & 1726,797 & 368 & 4,692 & & \\
\hline & 50 years and above & 82 & 12,01 & 2,169 & Toplam & 1743,315 & 370 & & & \\
\hline & Total & 371 & 11,93 & 2,171 & & & & & & \\
\hline \multirow[t]{4}{*}{ ÇB } & 39 yaş ve altı & 105 & 8,85 & 3,838 & G. Between & 76,929 & 2 & 38,464 & 3,368 & 036 \\
\hline & 40-49 yaş & 184 & 8,70 & 2,985 & G. in & 4203,061 & 368 & 11,421 & & \\
\hline & 50 yaş ve üz & 82 & 9,84 & 3,585 & Total & 4279,989 & 370 & & & \\
\hline & Toplam & 371 & 8,99 & 3,401 & & & & & & \\
\hline Genel & 39 yaş ve altı & 105 & 87,83 & 9,850 & G. Between & 418,790 & 2 & 209,395 & 2,526 & 081 \\
\hline \multirow[t]{3}{*}{ Ölçek } & 40-49 yaş & 184 & 90,12 & 8,009 & G. in & 30503,906 & 368 & 82,891 & & \\
\hline & 50 yaş ve üz & 82 & 88,26 & 10,349 & Total & 30922,695 & 370 & & & \\
\hline & Toplam & 371 & 89,06 & 9,142 & & & & & & \\
\hline
\end{tabular}

As shown in Table 15, an ANOVA test was used to determine the differences between school administrators' perceptions of school climate according to their age.

Table 16. Scheffe test results for determining the significant difference between school agents' age and teacher age groups

\begin{tabular}{|c|c|c|c|c|c|}
\hline Aspect of Average & Score (i) & Average Score (j) & $\bar{x}_{i}-\bar{x}_{j}$ & $S h_{\bar{x}}$ & $P$ \\
\hline \multirow[t]{6}{*}{ LEB } & 39 years and below & $40-49$ years &,- 835 & 487 & 231 \\
\hline & & 50 years and above & ,958 & ,587 & ,265 \\
\hline & $40-49$ years & 39 yaş ve altı &, 835 & ,487 & ,231 \\
\hline & & 50 yaş ve üz & $1,793^{*}$ & ,529 &, 003 \\
\hline & 50 years and above & 39 years and below &,- 958 & ,587 & ,265 \\
\hline & & $40-49$ years & $-1,793^{*}$ &, 529 & ,003 \\
\hline \multirow[t]{6}{*}{ ÇB } & 39 years and below & 40-49 years &, 147 & ,413 & ,939 \\
\hline & & 50 years and above &,- 994 & ,498 & ,138 \\
\hline & $40-49$ years & 39 years and below &,- 147 & ,413 & ,939 \\
\hline & & 50 years and above & $-1,140^{*}$ & ,449 &, 041 \\
\hline & 50 years and above & 39 years and below & ,994 & ,498 & ,138 \\
\hline & & $40-49$ years & $1,140^{*}$ & ,449 & 041 \\
\hline
\end{tabular}

Table 16 to determine the differences between school principals' school climate perceptions of teachers according to their age, Leadership and Interaction Size and Conflict Dimension to identify groups that have a significant difference in the Scheffe test was conducted and the results of the leadership and interaction in the sub-dimension 40- It was observed that the school principals of the age group of 40-49 years who had a significant difference between the 49 age group and the age group of 50 and above evaluated the school principals more positively, and 
the group which is 50 years of age and above evaluated more positively in the conflict dimension by the $40-49$ year olds.

Correlation Analysis Between Communication Competence and School Climate

Correlation Analysis between Communication Proficiency Scale and School Climate Results are given in Table 17.

Table 17. Results of the correlation analysis

\begin{tabular}{|c|c|c|c|c|c|c|c|c|c|c|c|c|}
\hline & & EYB & BDYB & EDYB & SRYB & Genel Ölçek & DOAB & LEB & BEB & SB & ÇB & General Scale \\
\hline \multirow[t]{3}{*}{ GIYYB } & $\mathrm{R}$ &, $860^{* *}$ &, $843^{* *}$ &, $793^{* *}$ &, $750^{* *}$ &, $942^{* *}$ &, $474^{* *}$ &, $673^{* *}$ &, $345^{* *}$ & $280^{* *}$ &,$- 269^{* *}$ &, $539^{* *}$ \\
\hline & $\mathrm{P}$ &, 000 &, 000 &, 000 &, 000 &, 000 &, 000 &, 000 &, 000 &, 000 &, 000 &, 000 \\
\hline & $\mathrm{N}$ & 371 & 371 & 371 & 371 & 371 & 371 & 371 & 371 & 371 & 371 & 371 \\
\hline \multirow[t]{3}{*}{ EYB } & $\mathrm{R}$ & 1 &, $827^{* *}$ &, $792^{* *}$ &, $782^{* *}$ &, $948^{* *}$ &, $471^{* *}$ & $688^{* *}$ &, $371^{* *}$ &, $294^{* *}$ &,$- 263^{* *}$ &, $556^{* *}$ \\
\hline & $\mathrm{P}$ & &, 000 &, 000 &, 000 &, 000 &, 000 &, 000 &, 000 &, 000 &, 000 &, 000 \\
\hline & $\mathrm{N}$ & & 371 & 371 & 371 & 371 & 371 & 371 & 371 & 371 & 371 & 371 \\
\hline \multirow[t]{3}{*}{ BDYB } & $\mathrm{R}$ & & 1 &, $778^{* *}$ &, $761^{* *}$ &, $921^{* *}$ &, $425^{* *}$ &, $641^{* *}$ &, $326^{* *}$ & $276^{* *}$ &,$- 288^{* *}$ &, $492^{* *}$ \\
\hline & $\mathrm{P}$ & & &, 000 &, 000 &, 000 &, 000 &, 000 &, 000 &, 000 &, 000 &, 000 \\
\hline & $\mathrm{N}$ & & & 371 & 371 & 371 & 371 & 371 & 371 & 371 & 371 & 371 \\
\hline \multirow[t]{3}{*}{ EDYB } & $\mathrm{R}$ & & & 1 &, $744^{* *}$ &, $884^{* *}$ &, $538^{* *}$ &, $724^{* *}$ &, $435^{* *}$ &, $329^{* *}$ &,$- 305^{* *}$ &, $608^{* *}$ \\
\hline & $\mathrm{P}$ & & & &, 000 &, 000 &, 000 &, 000 & ,000 &, 000 &, 000 &, 000 \\
\hline & $\mathrm{N}$ & & & & 371 & 371 & 371 & 371 & 371 & 371 & 371 & 371 \\
\hline \multirow[t]{3}{*}{ SRYB } & $\mathrm{R}$ & & & & 1 &, $860^{* *}$ &, $458^{* *}$ &, $678^{* *}$ &, $392^{* *}$ &, $356^{* *}$ &,$- 216^{* *}$ &, $584^{* *}$ \\
\hline & $\mathrm{P}$ & & & & &, 000 &, 000 &, 000 &, 000 &, 000 &, 000 &, 000 \\
\hline & $\mathrm{N}$ & & & & & 371 & 371 & 371 & 371 & 371 & 371 & 371 \\
\hline \multirow[t]{3}{*}{ Genel Ölçek } & $\mathrm{R}$ & & & & & 1 &, $512^{* *}$ &, $738^{* *}$ &, $400^{* *}$ &, $326^{* *}$ &,$- 292^{* *}$ &, $598^{* *}$ \\
\hline & $P$ & & & & & &, 000 &, 000 &, 000 & 000 &, 000 &, 000 \\
\hline & $\mathrm{N}$ & & & & & & 371 & 371 & 371 & 371 & 371 & 371 \\
\hline \multirow[t]{3}{*}{ DOAB } & $\mathrm{R}$ & & & & & & 1 &, $711^{* *}$ &, $699^{* *}$ &, $499^{* *}$ &,$- 462^{* *}$ &, $833^{* *}$ \\
\hline & $\mathrm{P}$ & & & & & & &, 000 &, 000 &, 000 &, 000 &, 000 \\
\hline & $\mathrm{N}$ & & & & & & & 371 & 371 & 371 & 371 & 371 \\
\hline \multirow[t]{3}{*}{ LEB } & $\mathrm{R}$ & & & & & & & 1 &, $594^{* *}$ &, $482^{* *}$ &,$- 382^{* *}$ &, $846^{* *}$ \\
\hline & $\mathrm{P}$ & & & & & & & & ,000 &, 000 &, 000 &, 000 \\
\hline & $\mathrm{N}$ & & & & & & & & 371 & 371 & 371 & 371 \\
\hline \multirow[t]{3}{*}{ BEB } & $\mathrm{R}$ & & & & & & & & 1 &, $505^{* *}$ &,$- 377^{* *}$ &, $775^{* *}$ \\
\hline & $\mathrm{P}$ & & & & & & & & &, 000 &, 000 &, 000 \\
\hline & $\mathrm{N}$ & & & & & & & & & 371 & 371 & 371 \\
\hline \multirow[t]{3}{*}{ SB } & $\mathrm{R}$ & & & & & & & & & 1 &,$- 324^{* *}$ &, $656^{* *}$ \\
\hline & $\mathrm{P}$ & & & & & & & & & &, 000 &, 000 \\
\hline & $\mathrm{N}$ & & & & & & & & & & 371 & 371 \\
\hline \multirow[t]{3}{*}{ ÇB } & $\mathrm{R}$ & & & & & & & & & & 1 &,$- 152^{* *}$ \\
\hline & $P$ & & & & & & & & & & &, 003 \\
\hline & $\mathrm{N}$ & & & & & & & & & & & 371 \\
\hline
\end{tabular}

In the result of the analysis which was conducted to determine the correlation between Communicative Competency and School Atmosphere, except in the aspect of conflict, in all of the subdimensions positive significant correlations were seen. It can be said that this result is coherent as the aspect of conflict consists of negative articles. When these results are taken into consideration it can be said that according to teachers' perceptions as school managers' communicative competencies increase so does the perception about school's atmosphere.

\section{Conclusion and Discussions}

The findings, which are acquired as a result of this research, were evaluated to show and explain the correlational analysis between competency of communication and school atmosphere by asssessing the teachers perceptions of school managers' levels of general communications. These perceptions about the levels were then assessed in terms of various demographic variables and school teachers' perceptions of school atmosphere's various demographic variables.

In assessment of teachers' perception of school managers' general communication levels in comparison with demographic variables: There was no significant differences found in teachers' perception of school managers' 
levels of general communication in the general scale and subdimension, in terms of the teachers' educations and their duties.

While there were significant differences in teachers' perceptions of school managers' Socialrelaxation competence among the levels of general communication in comparison with those teachers' genders, there were no significant differences found in the general scale and other subdimensions (Aspect of General Communication Competency, Aspect Emphaty Competency, Aspect of Affiliation/Support Competency, Aspect of Behavioral flexibility, Aspect of Socialrelaxation Competency, General Competency Aspect).

In terms of Socialrelaxation competency, it was seen that men are inclined to assess school managers more positively when compared to women. In a similar study, according to Gökkaya (2009): In terms of teachers' perceiving school manager's communicative skills, teachers' genders contributed to significant differentiations in emotions of this perception ( $\mathrm{t}=2,08 \mathrm{vep}=0,038)$. According to this, female teachers evaluate the emotional aspect of school teachers' communicative skills as more negative than male teachers do. According to Aylk (2016), it is seen that teachers perceived school managers' communicative skills the highest in "Socialrelaxation" aspect, followed respectively by "understanding-emphaty" and "support" aspects. On the other hand total sum of all the subdimensions of communicative skills was calculated as $X=3.94$. In the light of these findings it is appropriate to say that school managers' communicative skills are at good levels in the range of "most of the time". Since in our country the number of male school managers is higher than female school managers; men stated that school managers have positive socialrelaxation levels when they communicate with school managers.

While in terms of considering teachers' seniority, among the levels of general communication skills, in teachers' perception of school managers' socialrelaxation aspect there is no significant differences found, in all other subdimensions of the total scale, there were significant differences found. (Aspect of General Communication Skill, Aspet of Emphaty Competenc, Aspect of Affiliation/Support Competency, Aspect of behavioral flexibility Competency). It was seen that aforementioned difference is in seniorities of groups with 1-5 years, an in between seniorities of groups with 6-10 and 16 above while it was seen that groups having little seniorities evaluated school managers as more positive. In all subdimensions the result is seen to be present. In a similar study by Tunç (2015) it was seen that between school managers' communicative competencies and teachers' branch and their occupational seniority there is a significant difference. In the variable of occupational seniortiy, seniorities of 30 years and above cause a significant difference by having the smallest average.

In another study which was conducted by Casiadi (2017), it was seen that teachers' views vary in relation with school managers' communication styles that they adopt, according to teachers' period of service. According to the result, it is seen that differences between teachers are seen more with the teachers that have 1-5 years of service. Teachers having little seniority may have less opportunity of comparing as they don't get to work with different school managers. Teachers having little seniorities evaluate as more positive since they lack experience.

In terms of teachers' perceptions of school managers' general communication skills in comparison with these school managers' ages, it was found that in the total scale and all other subdimensions there is a significant difference. (Aspect of General Communicative Competency, Aspect of Competence of Emphaty, Aspect of affiliation/support Competency, Aspect of Behavioral Flexibility Competency, Aspect of Socialrelaxation Competency). According to school managers' ages, there was a significant difference found in teachers' perception of school managers' levels of general communication, in total scale and all other subdimensions. It was seen that in the aforementioned difference, there is a significant difference in the total scale between the group of 50 and above years and other groups. Moreoever it was also seen that individuals who are young evaluated school managers as more positive.

According to Şimşek (2003), teachers with seniority 6-10 years and 11-15 years and teachers with $3-4$ years and 56 years in their schools have likewise views on the relationship between school culture and communication skills of school principals. exhibit a downward trend. It is thought that the main reason for this is that teachers do not feel the need to give importance to educational and managerial variables as they were in previous periods because they have accepted them to school members. Therefore, it is seen that the above comments on the task and seniority support each other. Teachers with seniority between 16-20 years and above and the teachers whose terms of service are 7-8 years and above have similar tendencies towards the relationship between school culture and communication skills of school principals.

Young teachers may have high expectations of communication skills from school principals. Teachers who have just graduated from the Faculty may not have much knowledge and skills about communication processes at school. Teachers with a high level may have the opportunity to compare the communication skills of school principals because they work in different schools. 


\section{Evaluation of Teachers' Perceptions of School Climate by Various Demographic Variables}

When the relevant area scanned forms the basis of education of the majority of the work done in Turkey about climate on the primary (Barnes, 2007; Tahaoğlu, 2007; Akar, 2006; Aceti, 2006; Emeksiz, 2003; Süpç, 2000; Oktaylar, 1997; Dagli, 1996; Turner , 1996; Öztürk, 1995). It has been observed that the researches conducted in secondary education, especially in the general upper secondary school (Karataş, 2008; Günbay, 2003; Köksal, 1991; Peker, 1993; Ekşi, 2006), encompasses the views and perceptions of teachers about school climate. According to the tasks of teachers, there was a significant difference in the dimension of sincerity between school perceptions and no significant difference was found between the total scale and other sub-dimensions. (Democratization and Adoption Size, Leadership and Interaction Dimension, Factors of Success, Conflict Size). In the dimension of intimacy, the deputy principals evaluated the school climate more positively than the teachers. In another study, according to Sönmez (2016), it is important to show that the gender status of teachers differentiates their perceptions about the intimacy dimension of school climate. In this respect, female teachers perceive the school climate as more sincere than male teachers.

According to the gender, education and seniority of the teachers, there was no significant difference between the school climate perceptions and other sub-dimensions. (Democratization and Adoption Size, Leadership and Interaction Dimension, Success Factors Dimension, Sincerity Size, Conflict Size). Unlike this result, Sayg1l1 (2010) found that school climate scale scores showed a significant difference in favor of male teachers according to gender variable. It was found that there was a significant difference in favor of female teachers according to gender variable of Sincerity subscale scores of School Climate Scale. It was determined that there was a significant difference according to the education climate of school climate scale scores and the difference between the graduate education group and the other education group group in favor of the graduate education group. It was found that the School Climate Scale disagreement subscale scores showed a significant difference according to the occupational year variable, and this difference was found to be in favor of employees for less than 5 years and less than 5 years among employees working for 16 years or more.

While there was a significant difference between the school climate perceptions of teachers and school climate perceptions, there was no significant difference between the total scale and other sub-dimensions.There is a significant difference between the 40-49 age group in the leadership and interaction sub-dimension group with the age group of 50 years and over, they have seen their managers more confrontational.

Similar to these findings, Saygili (2010) found that the 51-60 age group and the 51-60 age group had higher scores among the 51-60 age group. It was determined that there was a significant difference according to the age variable of the school climate scale sincerity subscale scores, and the difference was in favor of 41-50 age group between the age group of 30 and six and $41-50$ age group.

\section{Correlation Analysis Between Communication Competence and School Climate}

As a result of the correlation analysis conducted to determine the correlation between communication competence and school climate, it was seen that there was a significant positive correlation in all sub-dimensions except conflict dimension. It can be said that this result is consistent because the conflict size is composed of negative articles. When these results are taken into consideration, it can be said that the perception of school climate has increased as the communication adequacy of school principals increased.

\section{Recommendations}

1) Detecting how much of the problems which ocur in schools stem from communicational problems and developement of appropriate strategies may help contribute in creating a healthy communicational environment in educational organizations.

2) At the levels of school managers' communicative skills, a cooperation must be established with the universities and they must be informed as communication is a two-way process.

3) Based on school managers' having communicative skills' being an important factor in schools' having positive atmosphere, in service trainings and seminars for improving school managers' communicative skills must be organized.

4) In terms of school atmosphere, it was found that in the aspect of sincerity there is significant difference of perception of school manager's assistants compared to teachers. In that sense it may be suggested that teachers and school managers organize social activities where they can spend time together to increase the sincerity (dining, family visits, football, volleyball or sport activities etc...) 
5) To create a positive school atmosphere school managers must communicate more with teachers who has much more seniorities.

6) School managers can create a positive school atmosphere by observing teachers' attitudes towards school's climate and their occupations.

7) School managers must gather periodically to brainstorm about manage executive issues, they must be able to improve communicative skills and must exchange ideas about creating a positive school atmosphere.

8) School managers and teachers must put effort to make the environment they are in a positive one and must take necessary precautions. For this condition to be evaluated they must periodically conduct meetings to make self-evaluation.

\section{References}

Acet, Ö. (2006). İlköğretim Okullarında Örgüt İklimi ile Karar Katılma Süreci Arasındaki İlişki. (Yayımlanmamış Yüksek Lisans Tezi). Dokuz Eylül Üniversitesi Eğitim Bilimleri Enstitüsü, İzmir.

Açıkalın, A. (1995). Toplumsal Kurumsal ve Teknik Yönleriyle Okul Yöneticiliği (No:10). İkinci Basım, Ankara: PEGEM Yayın.

Akar, A. (2006). İlköğretim Okullarında Öğretmen Algllarına Göre, Yöneticilerin Yöneticilik Becerilerine Göre Örgüt İklimine Katkısı (Yayınlanmamış Yüksek Lisans Tezi). Gazi Üniversitesi Eğitim Bilimleri Enstitüsü, Ankara.

Aydın, M. (1993). Çă̆daş Eğitim Denetimi. Ankara: Personel Eğitim Merkezi.

Ayık, A., \& Uzun, T. (2016). Mersin Üniversitesi Eğitim Fakültesi Dergisi. Mersin University Journal of the Faculty of Education, 12(2), 672-688.

Balcı, A. (1993). Etkili Okul, Uygulama ve Araştırma. Yavuz Dağııımı, Ankara.

Başaran. (2008). Örgütsel Davranış Insanın Üretim Gücü. Ankara: Ekinoks Basım Yayın Dağıtım.

Bursalığlu, Z. (1982). Eğitim yöneticisinin yeterlikleri. Ankara: Ankara Üniversitesi Eğitim Fakültesi.

Bursalığlu. (1991). Eğitimde yenileşme ve demokratik liderlik. Ankara Üniversitesi, Eğitim Bilimleri Fakültesi Dergisi, 24(2), 669-674.

Bursalığlu. (1999). Okul Yönetiminde yeni Yapı ve Davranış. On birinci Basım. Ankara: PEGEM Özel Eğitim ve Hizmetleri.

Bursalığlu. (2005). Okul Yönetiminde Yeni Yapı ve Davranış. 13. Baskı, Ankara: PegemA Yayıncılık.

Bursalığlu. (2013). Okul Yönetiminde Yeni Yapı ve Davranış. Ankara: Pegem Akademi Yayıncılık.

Bursalığlu. (2015). Okul Yönetiminde Yeni Yapı ve Davranıs. PegemA, Ankara.

Canlı, S. (2016). İп̈nü Üniversitesi Ĕgitim Bilimleri Enstitüsü Eğitim Bilimleri Ana Bilim Dalı Eğitim Yönetimi ve Denetimi Bilim Dall "Okul Müdürlerinin Öğretmenlere Güveninin Okul İklimine Etkisi". Doktora Tezi.

Casiadi, S. (2017). Öğretmenlerin Okul Müdürlerinin iletişim Becerileri ve Çatıma Yönetimi Konusunda Görüleri. Çanakkale On Sekiz Mart Üniversitesi Eğitim Bilimleri Enstitüsü Eğitim Bilimleri Anabilim Dalı Eğitim Yönetimi ve Denetimi Bilim Dalı Yüksek Lisans Tezi.

Çelik, Ç. (2007). İlköğretim Okulu Müdürlerinin İletişim Becerileri ile Tükenmişlik Düzeyleri Arasındaki İlişkisi (Gaziantep İli Merkez İlçeleri Örneği). Yüksek Lisans Tezi, Gazi Üniversitesi, Ankara.

Çelik, Ç. (2005). Liderlik. Eğitim ve Okul Yöneticiliği El Kitabı (Editör: Yüksel Özden). Ankara: PegemA Yayincilik.

Dağlı, A. (1996). İlköğretim Okullarının Örgüt İklimi Adana ve Gaziantep illeri örneği. Hacettepe Üniversitesi, Sosyal Bilimler Enstitüsü.

Deas, E. (1994). Board and Administration Relationships Contributingto Community College Climate: A Case Study. Community College Review, 22(1), 44-53.

Ekşi, F. (2006). Rehber Öğretmenlerin Okul İklimi Alglları ile Kaygı Düzeyleri Arasındaki İlişki Üzerine Bir Araştırma. Yayınlanmamış Yüksek Lisans Tezi, Marmara Üniversitesi Eğitim Bilimleri Enstitüsü, İstanbul.

Emeksiz, Ö. (2003). İlköğretim Okulu Öğretmenlerinin Okul İklimi ve Liderlik Etkenlerine İlişkin Görüşleri. Yayınlanmamış Yüksek Lisans Tezi, Yüksek Lisans Tezi Afyon Kocatepe Üniversitesi, Afyon. 
Gökkaya, S. (2009). Okul Müdürlerinin İletişim Becerilerinin Öğretmen Algılarına Göre İncelenmesi (Kırklareli İli Örneği).

Günbay, İ. (2003). School Climateandits Effects on High School Teachers in Different Teaching Categories. Sosyal Bilimler Dergisi, Haziran, V, 171-182.

Hoy, W. K. (2003). School climate. In J. W. Guhtrie (Ed.), Encylopedia of education (pp. 22-24). New York: Thompson Gale.

Karataş, S. (2008). Okul Müdürlerinin Etkililiği ve Okul İklimi. Yayınlanmamış Yüksek Lisans Tezi, Yeditepe Üniversitesi, Sosyal Bilimler Enstitüsü, İstanbul.

Kuperminc, G. P., Leadbeater, B. J., Emmons, C., \& Blatt, S. J. (1997). Perceived school climate and difficulties in the socialad justment of middle school students. Applied Developmental Science, 1(2), 76-88. https://doi.org/10.1207/s1532480xads0102_2

Oktaylar, H. C. (1997). Ağrı İlinde Görev Yapan İlkokul Öğretmenlerinin Çalıştıkları Okulun Örgütsel İklimini Algllama Düzeyleri. Yayınlanmamış Yüksek Lisans Tezi, Atatürk Üniversitesi Sosyal Bilimler Enstitüsü, Erzurum.

Özden, Y. (2013). Eğitimde yeni değerler. Ankara: Pegem Akademi Yayıncılık.

Öztürk, N. (1995). İlkokul Öğretmenlerinin Çalıştıkları Okulun İklimine İlişkin Alglları İle Gerilim Düzeyleri Arasındaki Illişskiler. Yayınlanmamış Yüksek Lisans Tezi. Sosyal Bilimler Enstitüsü, İzmir.

Peker, Ö. (1993). Okullarda örgütsel havanın çözümlenmesinde bir yöntem. Amme İdaresi Dergisi, 26(4), 21-43.

Peker, Ö. (1993). Okullarda Örgütsel Havanın Çözümlenmesinde Bir Yöntem Ankara: Türkiye Orta Doğu Amme İdaresi Dergisi, 26, 4.

Saygıl1, G. (2010). Öğretmenlerin Kişilik Özellikleri İle Okul İklimi Algıları Arasındaki İlişskinin İncelenmesi (İstanbul İli Sancaktepe İlçesi Örneği), Yüksek Lisans Tezi, Yeditepe Üniversitesi Sosyal Bilimler Enstitüsü.

Sönmez, E. (2016). Öğretmenlerin Okul İklimi Algllarının Çeşitli Demografik Değişkenlere Göre İncelenmesi: Bir Meta Analiz Çalı̧̧ması. Gazi Üniversitesi Eğitim Bilimleri Enstitüsü Eğitim Bilimleri Ana Bilim Dalı Yüksek Lisans Tezi.

Süpçin, E. (2000). Illköğretim Okullarının Örgüt İklimi. Yayınlanmamış Yüksek Lisans Tezi, Pamukkale Üniversitesi, Sosyal Bilimler Enstitüsü, Denizli.

Şahin, E. A. (2000). İlköğretim okulu müdürlerinin yeterlikleri. Kuram ve Uygulamada Eğitim Yönetimi Dergisi, 2, 243-260.

Şimşek, Y. (1997). Örgütsel İletişsimde Engel ve Bozukluklar: Anadolu Üniversitesi İletişim Bilimleri Fakültesinde Bir Uygulama. Yüksek Lisans Tezi.

Şimşek, Y. (2003). Okul Müdürlerinin İletişim Becerileri ile Okul Kültürü Arasındaki İlişsi (Eskişehir İli Örneği). Doktora Tezi, Anadolu Üniversitesi Eğitim Bilimleri Enstitüsü Eskişehir.

Şişman, M. (2004). Türk Eğitim Sistemi, Eğitim ve Okul Yöneticiliği El Kitabı. Ankara: Pegem A Yayıncılık.

Şişman, M. (2012). Eğitimde mükemmellik araylşı (Etkili okullar).Ankara: Pegem Akademi Yayıncılık.

Tahaoğlu, F. (2007). İlköğretim Okulu Müdürlerinin Liderlik Rollerinin Örgüt İklimi Üzerine Etkisi. Yayınlanmamış Yüksek Lisans Tezi, Gaziantep Üniversitesi Sosyal Bilimler Enstitüsü, Gaziantep.

Topluer, A. (2008). İn̈̈n̈̈ Üniversitesi Sosyal Bilimler Enstitüsü İlköğretim Okulu Yöneticilerinin İletişim Yeterlikleri ile Örgütsel Çatışma Düzeyi Arasındaki İlişki. Malatya İli Örneği. Yüksek Lisans Tezi.

Tuna, Z. (1996). Okul Müdürlerinin Yönetsel Davranışlarının Örgüt İklimine Etkisi. Yayınlanmamış Yüksek Lisans Tezi, Ankara Üniversitesi, Sosyal Bilimler Enstitüsü, Ankara.

Tunç R. (2015). Okul Yöneticilerinin Iletişim Yeterliliklerinin Öğretmenlerin Stres Düzeyleri Üzerindeki Etkilerinin İncelenmesi. Avrasya Üniversitesi Sosyal Bilimler Enstitüsü Yönetim Bilimleri Anabilim Dal.

Wallace, J., Hunt, J., \& Richards. (1996). The Relationship Between Organizational Cultere, Organizational Climate and Managerial Valuse. The International Journal of Public Sector Management, 12, 7. https://doi.org/10.1108/09513559910305339

Wei, L. T. (2003). Organizational climate and effectiveness in junior-middleschools in P. R. China. University of Regina. ProQuest Dissertationsand Theses, AAT MQ92858. 


\section{Copyrights}

Copyright for this article is retained by the author, with first publication rights granted to the journal.

This is an open-access article distributed under the terms and conditions of the Creative Commons Attribution license (http://creativecommons.org/licenses/by/4.0/). 